\title{
Demonstration of Transformable Manufacturing Systems through the Evolvable Assembly Systems Project
}

\author{
David Sanderson, Alison Turner, Emma Shires, Jack Chaplin, and Svetan Ratchev \\ Institute for Advanced Manufacturing, University of Nottingham
}

\begin{abstract}
Evolvable Assembly Systems is a five year UK research council funded project into flexible and reconfigurable manufacturing systems. The principal goal of the research programme has been to define and validate the vision and support architecture, theoretical models, methods and algorithms for Evolvable Assembly Systems as a new platform for open, adaptable, context-aware and cost effective production. The project is now coming to a close; the concepts developed during the project have been implemented on a variety of demonstrators across a number of manufacturing domains including automotive and aerospace assembly. This paper will show the progression of demonstrators and applications as they increase in complexity, specifically focussing on the Future Automated Aerospace Assembly Phase 1 technology demonstrator (FA3D). The FA3D Phase 1 demonstrated automated assembly of aerospace products using precision robotic processes in conjunction with lowcost reconfigurable fixturing supported by large volume metrology. This was underpinned by novel agent-based control for transformable batch-size-of-one production. The paper will conclude by introducing Phase 2 of the Future Automated Aerospace Assembly Demonstrator - currently in development - that will translate the Evolvable Assembly Systems research to a higher technology readiness level and address the challenges of scalable and transformable manufacturing systems.
\end{abstract}

\section{Introduction}

Assembly of final products in manufacturing sectors such as the automotive, aerospace, pharmaceutical, and food industries is a key production process in high labour cost areas such as the UK. To respond to the current challenges manufacturers need to transform current capital-intensive assembly lines into smart systems that can react to external and internal changes and can self-heal, self-adapt and reconfigure. This need is dictated by:

1. A demand for rapid ramp-up and downscale of production systems;

2. The fact that current assembly systems lack autonomous responsiveness to disruptive events and demand fluctuations; and

3. An economic and societal drive towards 'manufacturing as a service'.

Consequently, a need has been identified for a radically new approach towards the development of future assembly systems that are able to continuously evolve in response to changes in product requirements and demand. Such a system should take advantage of emerging new technologies, enable extremely short set-up times, and support a low cost of maintenance, system reconfiguration, and capability upgrade. As the level and type of automation changes, future assembly systems will also require a different type of engagement of human operators in hybrid decision-making, monitoring, and system adaptation.
The research programme delivered adaptable and cost effective manufacture predicated on complex collective adaptive manufacturing systems concepts [1]. Such concepts lead to extremely flexible and evolvable manufacturing infrastructure, providing production platforms with many of the "self-x" (sometimes called "self-*") properties [2,3], particularly self-configuration, selforganisation, and self-adaptation. These properties have been identified as key to the future vision of Cyber-Physical Production Systems (CPPS) [4,5] designed to give industry the ability to respond to and solve current and future societal grand challenges linked to retaining and expanding manufacturing operations in the UK [6].

The ultimate outcome of the Evolvable Assembly Systems (EAS) research is to enable a compressed product life cycle through the delivery of robust and compliant manufacturing systems that can be rapidly configured and optimised, thus reducing production ramp-up times and programme switchovers. Our approach of building an underlying architecture, using simulated and real-world data to test and populate models, and working closely with industry stakeholders, has ensured scalable and adaptable approaches that are transferable between different manufacturing sectors. Throughout the research programme, the techniques and results of the project have been demonstrated on a variety of demonstration production systems based at the University of Nottingham.

After a brief literature review to provide context of the field of flexible and reconfigurable manufacturing, this paper details the progression of the EAS project demonstrators. Each demonstrator in the project builds upon previous research, culminating in the FA3D and leading to the proposed FA3D2. The first project demonstrator was the Smart Manufacturing And Reconfigurable Technologies (SMART) demonstrator, which enhanced an existing discrete process training cell using the EAS agent-based control approach for customised pharmaceutical products. This section will also be used to introduce the basic technical concepts of the EAS approach. This was followed by enhancing the Precision Assembly Demonstrator (PAD), which added robotic assembly of non-structural sub-components for the automotive or aerospace industries. The Future Automated Aerospace Assembly Phase 1 demonstrator (FA3D) brought two robotic assembly cells: the flexible and reconfigurable FA3D-ABB (named for the brand of robots used in it) for component inspection and human-robot interaction, and the transformable FA3D-KUKA (likewise named) for precision assembly of aerospace assemblies using low-cost reconfigurable fixturing. Finally the paper will discuss the forthcoming Future Automated Aerospace Assembly Phase 2 demonstrator (FA3D2), which aims to build on the FA3D cells and the results of the EAS project to demonstrate truly scalable and transformable manufacturing for complete aerospace products.

\section{Background}

There is a significant body of research in reconfigurable manufacturing systems [7-9], automatic and adaptive control [1012], and manufacturing systems modelling and simulation [13-16]. The bionic and fractal factory concepts [17-19] proposed an 
integrated approach to manufacturing systems using an analogy with living organisms that try to adapt to changes at different levels of the manufacturing enterprise. Holonic Manufacturing Systems inspired by early complex systems research [20-23] use loosely coupled holons to represent physical or logical activities such as robots, machines, orders, or even factories that cooperate to achieve their goals [24]. Building on evolutionary computational theories new types of evolvable manufacturing systems have been proposed capable of optimising their performance in changing environments [25-28]. A concept founded on colonies of insects, swarm intelligence has been suggested as a method for collective systems adaptability, based on simple entities interacting locally with each other and with their environment [29-32]. Many approaches based around the concept of intelligent agents [33] have also been suggested [34-36] as a way to implement intelligent automation.

The concept of co-evolution of products, processes and production systems in response to evolving external drivers such as new materials, technologies, services, and communications has been a subject of debate [37]. Recent works have identified adaptability, changeability, self-resilience, self-improvement and co-creation as key facets of future responsive and flexible production systems $[4,5,38]$ - these properties are generally considered key to the various trends broadly described as "Industrie 4.0", "Digital Manufacturing", "Smart Manufacturing", or similar [39-45]. Achieving cohesive, coordinated, and balanced approach between products, processes, and systems in their continuous development and evolution is a key challenge for future successful and cost effective manufacture. This is particularly the case in high value industries, such as aerospace, and in economies where the cost of labour and materials is high. Evolution of a system can be triggered by different factors and driven by a variety of selective forces including system resilience to breakdowns, adaptation to changing product requirements, mutability of processes and equipment components, performance characteristics, and other indicators [37]. The EAS project aims to address these challenges through a CPPS-based approach to evolvability that can be applied to different manufacturing system levels such as devices, modules, workstations, cells, and systems.

\section{Smart Manufacturing And Reconfigurable Technologies (SMART)}

As the first demonstrator for the EAS project, the SMART demonstrator [46] was designed around one of the main project drivers: the trend towards ever more customised and personalised products. This is formalised in terms of the "batch size of one" (BSo1) problem. In other words, every product produced by the system may be unique within some set of constraints. This problem is relevant to a wide range of manufacturing domains: it is common in consumer products in terms of personalisation; is referred to as "mass-customisation" in automotive; has begun to attract interest in the food and pharmaceutical industries under the banners of "personalised food" and "personalised medicine" respectively; and is also applicable to the aerospace industry, both addressing the situation where customers (countries or companies) often require specific product variants, and where low production rates require low production volumes that approach the BSo1 problem. In EAS, the BSo1 problem is addressed through recipe-based production, in which each product is specified using a recipe file that contains all the required process information to produce the product. The specific application chosen for the SMART demonstrator was an abstracted personalised food or pharmaceuticals scenario.
The SMART demonstrator was designed to address the BSo1 problem and serves as an initial testbed for a number of other concepts that the EAS project would develop further. As such, it also demonstrates the application of recipe- and agent-based control and routing to a legacy modular production system. As part of this, fundamental disruption response could also be demonstrated.

Physically, the SMART demonstrator was based on an SMC Training HAS-200 [47], an automated system designed for use in training to demonstrate a variety of production concepts and enable training in PLC programming and operation. The system as-sold consists of a number of modular stations that can be combined in a variety of configurations around a conveyor belt to produce a large range of product variants. The product variants are all based around small plastic containers that are filled with different coloured pellets in different orders and amounts, then given a custom-printed label on their lid. Each station consists of the production hardware, a connection to the conveyor belt, and a Programmable Logic Controller (PLC) to control the hardware. All of the stations perform a small number of tightly constrained discrete processes.

As this demonstrator was not purchased specifically for the project, it was treated as a 'legacy' system that was to be enhanced with agentbased control to provide recipe-based BSo1 production. As can be seen in Figure 1, the eight stations that comprise the demonstrator in our case are:

1. Loading station: containers are loaded from a magazine to the conveyor belt.

2. Red pellet filling station: fills containers with red pellets, and can also load specific red-only containers to the conveyor.

3. Blue pellet filling station: fills containers with blue pellets, and can also load specific blue-only containers to the conveyor.

4. Yellow pellet filling station: fills containers with yellow pellets, and can also load specific yellow-only containers to the conveyor.

5. Testing station: the containers are tested against the expected fill depth of pellets using a linear encoder.

6. Testing station: the containers are tested against the expected fill depth of pellets using a linear potentiometer.

7. Lidding \& labelling station: the containers have a lid fitted to them, and a custom-printed label applied.

8. Palletisation station: the completed containers are removed from the conveyor belt and added to a pallet for delivery.

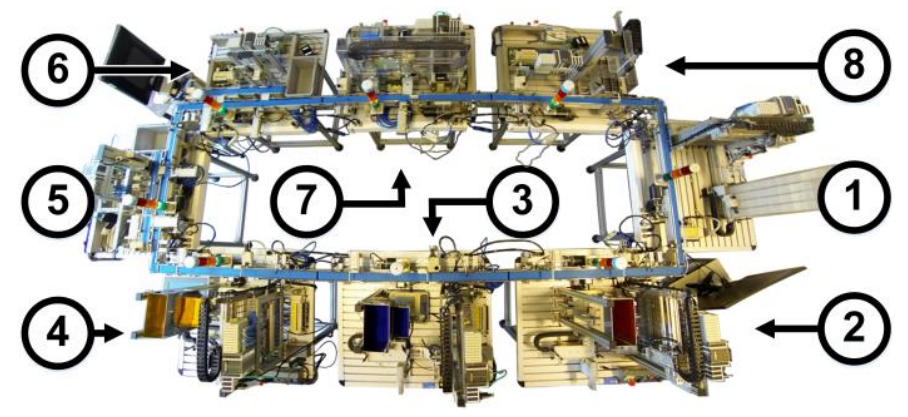

Figure 1. The SMART demonstrator shown from above with labels indicating modules.

For the purposes of the EAS project, each station PLC is connected to a low-cost embedded computer running an intelligent agent [33]. In 
the case of the SMART demonstrator, Raspberry Pi 3 Model B embedded computers [48] were used, but other options are available. While the PLC at each station retains low-level control, the corresponding agent is responsible for higher-level control, communicating with the rest of the system, and maintaining both a context-aware view of the wider system and a set of tasks required to achieve production goals. The multi-agent system controls and coordinates the whole production system and orders can be submitted by an operator or customer through a network-connected interface. The high-level agent-oriented architecture for EAS is shown in Figure 2 from a system-level viewpoint. On the left-hand side is the individual agent-resource: each agent maintains a local internal model of the production system and communicates with both its own resource and with other agents. The middle of the diagram represents the joint data model formed from the collective knowledge of all agents. This knowledge can be used for system-level functions like reconfiguration and planning. The right-hand side shows that the system is situated in the wider environment. The system interfaces with the overall enterprise to receive additional context.

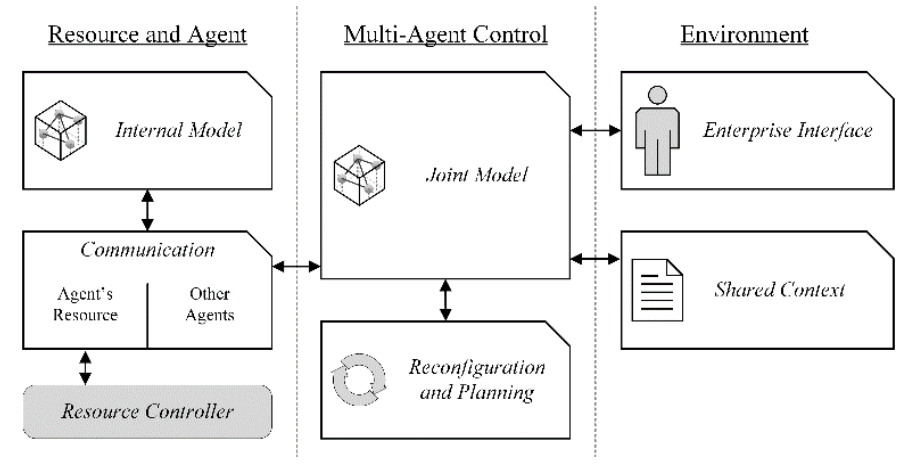

Figure 2. High-level system view of the EAS architecture.

In implementation terms, communication between agents is handled by a Data Distribution Service (DDS) [49] operating over a WiFi network. The DDS provides a standards-based publish-subscribe communication channel for the system. The generic high-level system stack for EAS is shown in Figure 3, depicting a single agentresource pairing. The agents run in Java using JADE, an agent development framework [50], and communicate to the resource controller through a translation layer and with each other via DDS. While most agents control a physical production resource, some agents provide interfaces with the wider enterprise or with a human operator.

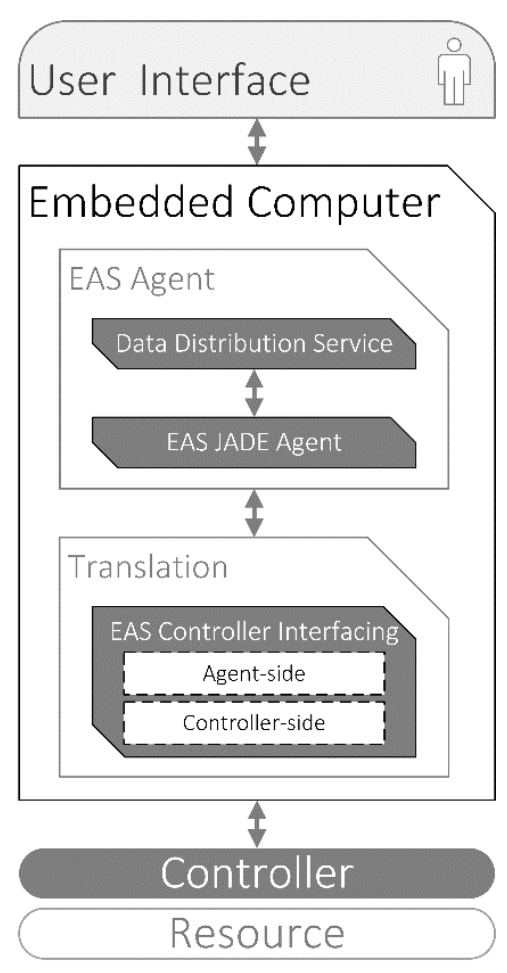

Figure 3. Generic EAS system stack for a single agent-resource pairing.

The system interface allows the user to specify a custom recipe for a product, which can then be produced on the system. When a recipe is submitted, the agent system first attempts to check whether the recipe can be completed with the existing capabilities in the system, and - if so - how the tasks should be allocated to each station and the required route for the product through the system. These two questions of whether and how a product can be manufactured with a specific set of resources are the manufacturability and control problems of BSo1 production ${ }^{1}$. Briefly, the system and the submitted recipe are represented internally as labelled transition systems. An adapted simulation relation is used to check manufacturability, followed by a controller synthesis process to generate the control solution. Further details can be found in $[51,52]$.

As the first demonstrator for the EAS project, the SMART demonstrator acts as a testbed for the overall architectural concepts, as well as successfully proving out the recipe- and agent-based BSo1 production approach in the context of discrete constrained processes. The application of this approach to legacy systems and the fundamentals of disruption response are also demonstrated. A video of the SMART demonstrator can be found online at [53].

\section{Precision Assembly Demonstrator (PAD)}

While the SMART demonstrator shows a set of tightly constrained discrete processes, the PAD [54] extends the application of EAS concepts to small robotic assembly tasks. In this case for small-

\footnotetext{
${ }^{1}$ In our other papers, the "manufacturability" problem is sometimes referred to as the "realisability" problem. These two terms can be considered interchangeable.
} 
batches of consumer products or for assembly of components for the automotive or aerospace industries; the specific exemplar product was the detent hinge of a glove compartment from a truck.
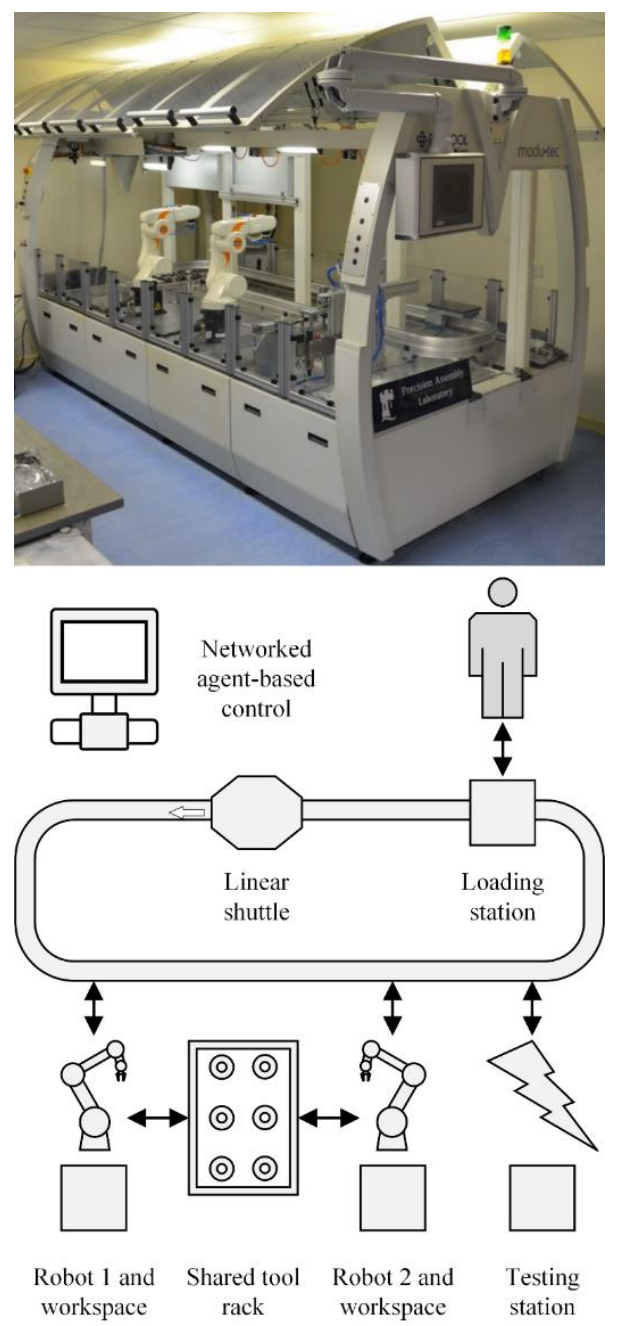

Figure 4. The PAD and layout.

As shown in Figure 4, the PAD is based on a Feintool Modutec platform where each cabinet in the station holds a resource. In this case there are: two robot arms that each have their own workspace; an automatic tool changer shared by the two robots, with a variety of tools that are tracked with RFID tags; a testing station that performs a force test and a vision test; a loading station; and a linear shuttle that transports pallets between each station. Each cabinet is connected to the main platform through a single connector cable, allowing them to be quickly and easily unplugged or plugged in.

The PAD also demonstrates recipe-based BSo1 production, and utilises an agent-based planning and control system, but focusses more on planning - rather than routing - and also provides the opportunity to demonstrate the concept of "plug and produce"2 . In

${ }^{2}$ Similar to the concept of "plug and play" for computers (which is now commonplace due to the proliferation of USB connections) but for manufacturing resources.

Page 4 of 10 physical terms this is accomplished through the integration of a common set of hardware and software interfaces into the modular cabinets that make up the demonstrator. The software implementation of the plug and produce functionality is provided by modification of the labelled transition systems that represent the system. As the system structure is modified, the manufacturability and control algorithms re-run and re-plan taking the new system configuration into account.

The PAD builds on the SMART demonstrator by introducing robotic assembly and acts as a validation by applying the approaches to another system and assembly domain.

\section{Future Automated Aerospace Assembly Demonstrator (FA3D)}

While the previous two demonstrators focus on small parts and products, the FA3D brings EAS to aerospace assembly operations. In response to the industrial challenges of increased automation, lower batch sizes, and shorter product lifecycles, the FA3D uses recipeoriented and agent-based control to enable a flexible, reconfigurable, and "transformable" approach to high-accuracy aerospace assembly across two production cells. The transformable approach relies on a cyber-physical systems concept of production systems in which all entities have a digital representation, communicate with each other, and can transform their configuration to meet the current system requirements.

\section{$F A 3 D-A B B$}

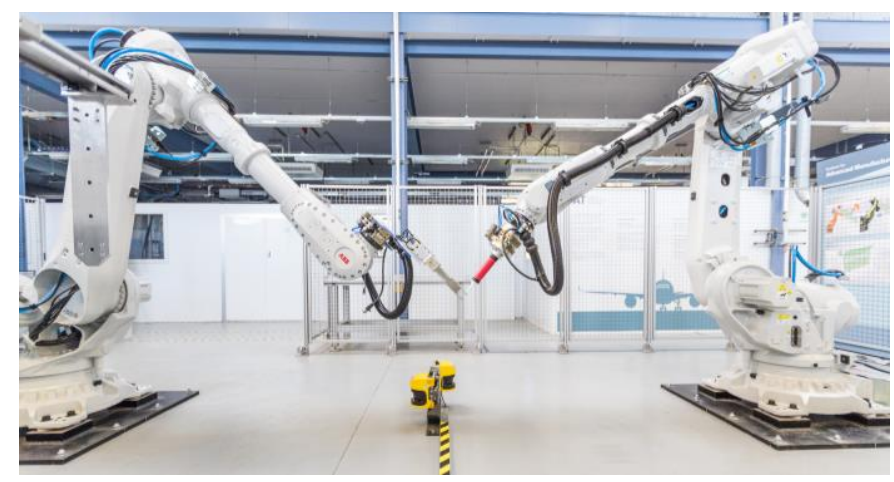

Figure 5. The FA3D-ABB cell.

The first of these cells, the FA3D-ABB [55] shown in Figure 5, consists of two ABB IRB6700 robots, a tool rack for each robot, a shared central workspace, a part-loading conveyor, and a part storage rack. The conveyor and storage rack are accessible by one robot which holds parts, and the second robot performs operations on the held part. Each robot has access to a number of different end effectors on their respective rack and is equipped with an automatic tool changer. Similarly to the SMART, each resource is controlled by an agent deployed on a Raspberry Pi 3 Model B embedded computer connected to the relevant PLC. These resources are used to perform processes around the assembly and inspection of aerospace components as defined through a variety of recipe files. The recipe files are composed of a set of parameterised commands from the following list:

- Load and unload parts via conveyor, identifying them from RFID tags. 
- $\quad$ Pick, place, and manipulate a variety of trailing edge ribs and non-structural skin panels from a common single-aisle aircraft.

- Apply sealant.

- $\quad$ Store parts in rack for curing.

- Scan parts with a line scanner.

- Apply temporary fasteners (semi-manual process).

The cell layout is shown in Figure A 1, and includes an "ABB SafeMove zone"; when a human enters this zone the robots move at a reduced speed, and if a human moves beyond the zone into the main cell area, the robots go into a soft stop. This allows operations to resume once the cell is clear, in contrast to a hard stop safety system. Our approach allows the integration of manual operations where appropriate into the production process; in addition to the production resources there is also a network-attached human-machine interface (HMI) through which an operator can supervise and interact with the cell.

\section{FA3D-KUKA}

Where the FA3D-ABB focusses on aerospace components and subassemblies, the FA3D-KUKA [56] demonstrates assembly processes targeted at the scale of wingbox and small fuselage section. Building on the technologies from the EAS demonstrators that went before it, the cell is designed to demonstrate high-accuracy automated aerospace assembly utilising robotic automation, low-cost flexible fixturing, large volume metrology, and RFID part location and tracking in a transformable manner. Shown in Figure 6 assembling a demonstration wingbox, the cell consists of two KUKA KR270 robots and one KUKA KR1000 Titan robot. Each robot has a tool changer mounted on it. The KR270s each have access to a tool rack for the process end effectors which need to change frequently. As the Titan is only used for part positioning where the end effectors are larger and less frequently changed, they are changed manually. Available end effectors include:

- Drill with automatic extraction.

- Solid rivet gun capable of installing three sizes of rivet.

- Counter-pressure tool with changeable heads for drill and rivet operations.

- Small general-purpose pneumatic gripper.

- Variety of robot-side component positioning tools.

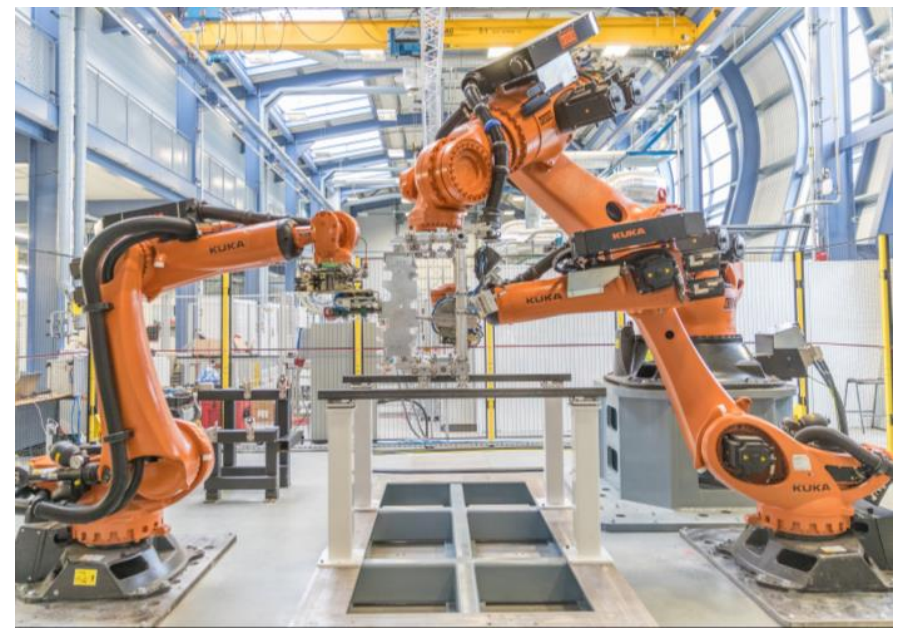

In addition to the process end effectors, all robots in the cell are connected to a photogrammetry system designed to improve their absolute positional accuracy to better than $0.3 \mathrm{~mm}$. This system adds an adaptive control loop around the movement commands in the robot controller. A camera array is used to correct the robot position based on static measurements of infra-red LEDs mounted to the fixtures and tools; more details of this system can be found in [56].

Mounted above the robot working area are an array of RFID sensors. These sensors are used to detect the (low precision) position of RFID tags that can be attached to all end effectors, fixtures, tools, and significant parts. High precision production operations can be verified through the use of a large-volume non-contact metrology solution based on a coherent laser radar providing accuracy of better than $0.3 \mathrm{~mm}$. The radar also includes a weather station used to monitor environmental conditions, including temperature and humidity.

All the resources in the system are connected via a hybrid Manufacturing-Enterprise Service Bus (MESB) using the EAS DDS approach. All of the data generated by the resources in the cell can be transmitted across the MESB as required. These can then be collected in a central database, providing the "product DNA" of the product being assembled - this is analogous to the concept of a "digital thread" [57] which stores all relevant information about the product throughout the lifecycle in a standardised digital format. By collating the low-precision RFID positional data, high-precision inspection data, environmental readings, and robot end effector locations in one place, the performance of the whole system can be better analysed.

The MESB is also the route by which the cell is controlled. Each PLC and controller in the cell is connected the MESB and provides a programme interface for each parameterised operation it can perform. This allows the cell to be quickly transformed for each new product and controlled according to the product recipe files as described above.

The final aspect of the FA3D-KUKA is a focus on low-cost reconfigurable fixturing. A variety of approaches were trialled to examine the trade-off between cost, reconfigurability, and rigidity of end effectors. The cheapest and most reconfigurable approach was a set of modular part positioning tools constructed from extruded aluminium; although very cheap and lightweight, these were unstable and posed a number of challenges for high-accuracy positioning operations and maintaining stability. At the opposite end of the spectrum, a bespoke welded steel positioner was constructed for one specific high-value component. Although it provided far better performance in terms of stability, the tool was correspondingly more expensive, and so heavy as to be only suitable for the Titan. Visible in the centre of Figure 6 is a set of DESTACO SpiderGrip [58], which was only used for public demonstrations using custom IP-free representative demonstration components.

\section{Combined FA3D System}

Both FA3D cells are designed so that they can function as a single coherent system by connecting both to the same hybrid MESB. In this case, the FA3D-ABB performs component-level preparation and inspection, before feeding the parts into the FA3D-KUKA for final assembly. A schematic of the combined control system is shown in Figure A 2. 
The FA3D cells show the application of the EAS concepts to a larger scale of assembly, in particular to aerospace assembly. As well as showing how the BSo1 approach can be implemented in a flexible and reconfigurable manufacturing system, the cells incorporate a much larger variety of integrated subsystems. While the agents in the SMART and PAD each control a self-contained station, in the FA3D the agents may be controlling a robot or a conveyor belt, interfacing with a metrology system, or providing an HMI for an operator.

\section{Future Automated Aerospace Assembly Demonstrator Phase 2 (FA3D2)}

Currently in the tendering phase, the FA3D2 is funded by the UK Industrial Strategy Challenge Fund [59]. The project aims to deliver a national experimental testbed and technology demonstrator in digitaland informatics-enabled aerospace manufacturing technologies. The intention is to provide opportunities for aerospace manufacturing businesses to test, demonstrate, and accelerate the implementation of new breakthrough technologies, thus allowing them to better compete on productivity, quality, and cost.

The FA3D2 programme builds on the core principles of flexible and reconfigurable assembly demonstrated throughout the EAS project to develop a solution that is transformable and scalable in both working volume (size) and production volume (rate). This is based on a framework for adaptable joining, metrology, and testing processes integrated into a common platform that can adapt to changing product requirements, insertion of new disruptive technologies, and respond in a cost-effective manner to product and rate variations.

Through working closely with the UK aerospace industry, several use cases have been developed that embody the range of industrial challenges within future manufacturing. These use cases are based around wing, nacelle and fuselage, and form a key input to the specification of the demonstrator platform. A number of enabling digital and manufacturing technologies have been identified that will ensure that experimental platform addresses the industrial requirements captured and advances the state of the art. An overview of the concept is shown in Figure A 3.

The FA3D2 project launched in January 2018 and is in the requirement capture and specification generation phase. As shown in Figure 7, the first phase of the project focusses on the reconfigurable, metrology enabled cell infrastructure and underpinning digital technologies. Following on from that will be the development of automated processes, intelligent fixturing and human centred production. The platform will then be utilised from 2021 to deliver impact through industrially focused research projects.

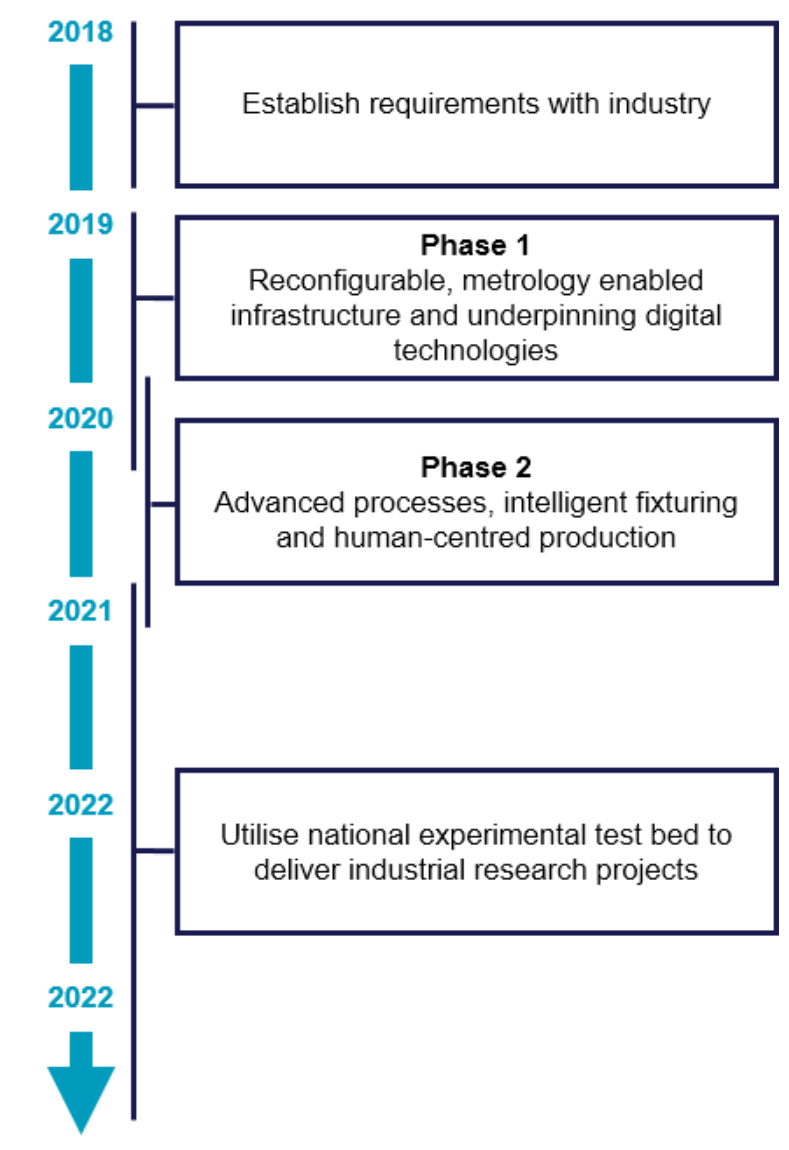

Figure 7. FA3D2 Project Timeline.

\section{Summary/Conclusions}

Through the described demonstrators, the EAS project has demonstrated a variety of applications for a newly developed platform for open, adaptable, context-aware and cost effective production. This EAS approach is based on cyber-physical systems concepts, reflecting current thinking as typified by Industrie 4.0 and similar programmes. The project has informed the development of the FA3D2, a forthcoming UK testbed for scalable and transformable aerospace manufacturing that leverages digital- and informaticsenabled technologies. A summary of all demonstrators discussed in this paper is given in Table A 1 .

\section{References}

1. Sanderson, D., Antzoulatos, N., Chaplin, J.C., Busquets, D., Pitt, J., German, C., Norbury, A., Kelly, E., and Ratchev, S., "Advanced Manufacturing: An Industrial Application for Collective Adaptive Systems," 2015 IEEE International Conference on Self-Adaptive and Self-Organizing Systems Workshops, IEEE, ISBN 978-1-4673-8439-1: 61-67, 2015, doi:10.1109/SASOW.2015.15.

2. Seebach, H., Ortmeier, F., and Reif, W., "Design and construction of organic computing systems," 2007 IEEE Congress on Evolutionary Computation, IEEE, ISBN 978-14244-1339-3: 4215-4221, 2007, doi:10.1109/CEC.2007.4425021. 
3. Onori, M., Semere, D., and Lindberg, B., "Evolvable systems: an approach to self-X production," Int. J. Comput. Integr. Manuf. 24(5):506-516, 2011, doi:10.1080/0951192X.2011.566282.

4. Rosen, R., Wichert, G. von, Lo, G., and Bettenhausen, K.D., "About The Importance of Autonomy and Digital Twins for the Future of Manufacturing," IFAC-PapersOnLine 48(3):567572, 2015, doi:10.1016/j.ifacol.2015.06.141.

5. Monostori, L., "Cyber-physical Production Systems: Roots, Expectations and R\&D Challenges," Procedia CIRP 17:9-13, 2014, doi:10.1016/j.procir.2014.03.115.

6. Technology Strategy Board, "A Landscape for the Future of High Value Manufacturing in the UK," 2012.

7. Koren, Y., Heisel, U., Jovane, F., Moriwaki, T., Pritschow, G., Ulsoy, G., and Brussel, H. Van, "Reconfigurable Manufacturing Systems," CIRP Ann. - Manuf. Technol. 48(2):527-540, 1999, doi:10.1016/S0007-8506(07)63232-6.

8. Mehrabi, M.G., Ulsoy, A.G., and Koren, Y., "Reconfigurable manufacturing systems: Key to future manufacturing," J. Intell. Manuf. 11(4):403-419, 2000, doi:10.1023/A:1008930403506.

9. Koren, Y., "Reconfigurable Manufacturing and Beyond," CIRP 3rd International Conference on Reconfigurable Manufacturing, 2005.

10. Monostori, L., Csáji, B.C., Kádár, B., Pfeiffer, A., Ilie-Zudor, E., Kemény, Z., and Szathmári, M., "Towards adaptive and digital manufacturing," Annu. Rev. Control 34(1):118-128, 2010, doi:10.1016/j.arcontrol.2010.02.007.

11. Müller-Schloer, C., Schmeck, H., and Ungerer, T., eds., "Organic Computing - A Paradigm Shift for Complex Systems," Springer Basel, 2011.

12. Parashar, M. and Hariri, S., "Autonomic Computing: Concepts, Infrastructure, and Applications," CRC press, 2007.

13. ElMaraghy, H., AlGeddawy, T., and Azab, A., "Modelling evolution in manufacturing: A biological analogy," CIRP Ann. - Manuf. Technol. 57(1):467-472, 2008, doi:10.1016/j.cirp.2008.03.136.

14. Choi, K.-H., Bae, C.-H., and Lee, S.-H., "Behaviour modelling and control of computer integrated manufacturing," Int. J. Comput. Integr. Manuf. 16(2):128-139, 2003, doi:10.1080/713804984.

15. Monostori, L. and Prohaszka, J., “A Step towards Intelligent Manufacturing: Modelling and Monitoring of Manufacturing Processes through Artificial Neural Networks," CIRP Ann. Manuf. Technol. 42(1):485-488, 1993, doi:10.1016/S00078506(07)62491-3.

16. Ueda, K., Vaario, J., and Ohkura, K., "Modelling of Biological Manufacturing Systems for Dynamic Reconfiguration," CIRP Ann. - Manuf. Technol. 46(1):343-346, 1997, doi:10.1016/S0007-8506(07)60839-7.

17. Warnecke, H.J., "Fractal Company - A Revolution in Corporate Culture," 1st ed., Springer Publishing Company, Incorporated, ISBN 978-3-642-55776-7, 2003, doi:10.1007/978-3-642-55776-7_3.

18. Tharumarajah, A., "Comparison of the bionic, fractal and holonic manufacturing system concepts," Int. J. Comput. Integr. Manuf. 9(3):217-226, 1996, doi:10.1080/095119296131670.

19. Kádár, B., Terkaj, W., and Sacco, M., "Semantic Virtual Factory supporting interoperable modelling and evaluation of production systems," CIRP Ann. - Manuf. Technol. 62(1):443446, 2013, doi:10.1016/j.cirp.2013.03.045.

20. Koestler and Arthur, The ghost in the machine., 1968.

21. Brussel, H. Van, Wyns, J., Valckenaers, P., Bongaerts, L., and Peeters, P., "Reference architecture for holonic manufacturing systems: PROSA," Comput. Ind. 37(3):255-274, 1998, doi:10.1016/S0166-3615(98)00102-X.

22. Leitão, P. and Restivo, F., "ADACOR: A holonic architecture for agile and adaptive manufacturing control," Comput. Ind. 57(2):121-130, 2006, doi:10.1016/j.compind.2005.05.005.

23. Babiceanu, R.F. and Chen, F.F., "Development and Applications of Holonic Manufacturing Systems: A Survey," J. Intell. Manuf. 17(1):111-131, 2006, doi:10.1007/s10845-0055516-y.

24. Sugi, M., Maeda, Y., Aiyama, Y., Harada, T., and Arai, T., “A holonic architecture for easy reconfiguration of robotic assembly systems," IEEE Trans. Robot. Autom. 19(3):457-464, 2003, doi:10.1109/TRA.2003.810241.

25. Leitão, P., Barbosa, J., and Trentesaux, D., "Bio-inspired multiagent systems for reconfigurable manufacturing systems," Eng. Appl. Artif. Intell. 25(5):934-944, 2012, doi:10.1016/j.engappai.2011.09.025.

26. Onori, M., Barata, J., and Frei, R., "Evolvable Assembly Systems Basic Principles," Information Technology For Balanced Manufacturing Systems, Springer US, ISBN 978-0387-36590-9: 317-328, 2006, doi:10.1007/978-0-387-365947_34.

27. Semere, D., Onori, M., Maffei, A., and Adamietz, R., "Evolvable assembly systems: coping with variations through evolution," Assem. Autom. 28(2):126-133, 2008, doi:10.1108/01445150810863707.

28. Neves, P. and Barata, J., "Evolvable production systems," Assembly and Manufacturing, 2009, IEEE International Symposium on, 189-195, 2009, doi:10.1109/ISAM.2009.5376907.

29. Park, H.-S. and Tran, N.-H., "An autonomous manufacturing system based on swarm of cognitive agents," J. Manuf. Syst. 31(3):337-348, 2012, doi:10.1016/j.jmsy.2012.05.002.

30. Dorigo, M., Bonabeau, E., and Theraulaz, G., "Ant algorithms and stigmergy," Futur. Gener. Comput. Syst. 16(8):851-871, 2000, doi:10.1016/S0167-739X(00)00042-X.

31. Bonabeau, E., Dorigo, M., and Theraulaz, G., "Swarm Intelligence: From Natural to Artificial Systems," Oxford University Press, Inc., ISBN 0195131592, 1999.

32. Marsh, L. and Onof, C., "Stigmergic epistemology, stigmergic cognition," Cogn. Syst. Res. 9(1-2):136-149, 2008, doi:10.1016/j.cogsys.2007.06.009.

33. Wooldridge, M. and Jennings, N.R., "Intelligent agents: Theory and practice," Knowl. Eng. Rev. 10(2):115-152, 1995.

34. Parunak, H.V.D., "Manufacturing Experience with the Contract Net," Distrib. Artif. Intell. 1:285-310, 1987.

35. Jones, A.T., Romero, D., and Wuest, T., "Modeling agents as joint cognitive systems in smart manufacturing systems," Manuf. Lett. 17:6-8, 2018, doi:10.1016/J.MFGLET.2018.06.002.

36. Antzoulatos, N., Castro, E., Silva, L. de, and Ratchev, S., "Interfacing Agents with an Industrial Assembly System for 'Plug and Produce': (Demonstration)," Proceedings of the 2015 International Conference on Autonomous Agents and Multiagent Systems, International Foundation for Autonomous Agents and Multiagent Systems, Richland, SC, ISBN 978-14503-3413-6: 1957-1958, 2015.

37. Tolio, T., Ceglarek, D., ElMaraghy, H.A., Fischer, A., Hu, S.J., Laperrière, L., Newman, S.T., and Váncza, J., "SPECIES --Co-evolution of products, processes and production systems," CIRP Ann. - Manuf. Technol. 59(2):672-693, 2010, doi:10.1016/j.cirp.2010.05.008.

38. Zuehlke, D., "SmartFactory --- Towards a factory-of-things," Annu. Rev. Control 34(1):129-138, 2010, doi:10.1016/j.arcontrol.2010.02.008. 
39. Bundesministerium für Bildung und Forschung, "Industrie 4.0 Innovationen für die Produktion von morgen, " 2014.

40. Kagermann, H., Helbig, J., Hellinger, A., and Wahlster, W., "Recommendations for Implementing the Strategic Initiative INDUSTRIE 4.0: Securing the Future of German Manufacturing Industry; Final Report of the Industrie 4.0 Working Group," 2013.

41. The Digital Manufacturing and Design Innovation Institute, "Strategic Investment Plan, 2015," http://dmdii.uilabs.org/, accessed Dec. 2018.

42. Industrial Value Chain Initiative, "Connected Manufacturing, 2014," https://www.iv-i.org/en/, accessed Dec. 2018.

43. Manufuture, "A Vision for 2020, Assuring the Future of Manufacturing in Europe," 2004.

44. European Chamber of Commerce in China, "China Manufacturing 2025: Putting Industrial Policy Ahead of Market Force," 2017.

45. Widell, H. and Lundberg, K., "Produktion2030 Agenda - Make in Sweden 2030," 2016.

46. Sanderson, D., Chaplin, J.C., Silva, L. De, Holmes, P., and Ratchev, S., "Smart Manufacturing and Reconfigurable Technologies: Towards an Integrated Environment for Evolvable Assembly Systems," 2016 IEEE 1st International Workshops on Foundations and Applications of Self* Systems (FAS*W), IEEE, ISBN 978-1-5090-3651-6: 263-264, 2016, doi:10.1109/FAS-W.2016.61.

47. SMC Training, http://www.smctraining.com/, accessed Dec. 2018.

48. Raspberry Pi 3 Model B,

https://www.raspberrypi.org/products/raspberry-pi-3-model-b/, accessed Dec. 2018.

49. Object Management Group, "Data Distribution Service", http://www.omg.org/spec/DDS/Current, accessed Dec. 2018.

50. Bellifemine, F., Poggi, A., and Rimassa, G., "JADE --- A FIPA-compliant agent framework," Proc. Fourth Int. Conf. Pract. Appl. Intell. Agents Multi-Agent Technol., 1999.

51. Silva, L. De, Felli, P., Chaplin, J.C., Logan, B., Sanderson, D., and Ratchev, S., "Realisability of production recipes," ISBN 9781614996712, 2016, doi:10.3233/978-1-61499-672-9-1449.

52. Silva, L. de, Felli, P., Chaplin, J.C., Logan, B., Sanderson, D., and Ratchev, S., "Synthesising Industry-Standard Manufacturing Process Controllers," Proceedings of the International Joint Conference on Autonomous Agents and Multiagent Systems, AAMAS, International Foundation for Autonomous Agents and Multiagent Systems, ISBN 9781510855076: 1811-1813, 2017.

53. Youtube, “"EPSRC Evolvable Assembly Systems SMART Demonstrator,"” https://youtu.be/9sQRYxLk5CE, accessed Dec. 2018.

54. Bakker, O.J., Chaplin, J.C., Silva, L. de, Felli, P., Sanderson, D., Logan, B., and Ratchev, S., "Toward Process Control from Formal Models of Transformable Manufacturing Systems," Procedia CIRP 63:521-526, 2017, doi:10.1016/j.procir.2017.03.159.

55. Sanderson, D., Chaplin, J., and Ratchev, S., "Common shared system model for Evolvable Assembly Systems," Model-Based Enterprise Summit 2018, NIST, 2018.

56. Drouot, A., Irving, L., Sanderson, D., Smith, A., and Ratchev, S., "A Transformable Manufacturing Concept for Low-Volume Aerospace Assembly," IFAC-PapersOnLine 50(1):5712-5717, 2017, doi:10.1016/j.ifacol.2017.08.1123.

57. Kinard, D., "Digital Thread and Industry 4.0," NIST Model Based Enterprise Summit, 2018.

58. DESTACO, https://www.destaco.com/, accessed Dec. 2018.

59. UK Industrial Strategy Challenge Fund, https://www.ukri.org/innovation/industrial-strategy-challengefund/, accessed Dec. 2018.

\section{Contact Information}

Dr David Sanderson,

Centre for Aerospace Manufacturing, University of Nottingham,

Unit 4, Easter Park,

Lenton Lane,

Nottingham, UK

NG1 1AT

Email: David.Sanderson@nottingham.ac.uk

\section{Acknowledgments}

The authors gratefully acknowledge the support provided by the UK EPSRC under Evolvable Assembly Systems (EP/K018205/1) and by Innovate UK under FA3D2 (reference 113163).

Published by SAE International as SAE Technical Paper 2019-011363, 2019, doi:10.4271/2019-01-1363.

\section{Definitions/Abbreviations}

FA3D

EAS

SMART

PAD

FA3D2

CPPS

BSo1

PLC

DDS

MESB
Future Automated Aerospace Assembly Demonstrator

Evolvable Assembly Systems

Smart Manufacturing And Reconfigurable

Technologies

(Demonstrator)

Precision Assembly Demonstrator

Future Automated Aerospace Assembly Demonstrator Phase 2

Cyber-Physical Production System(s)

Batch Size of One

Programmable Logic Controller

Data Distribution Service

Manufacturing-Enterprise Service Bus

Page 8 of 10 


\section{Appendix}

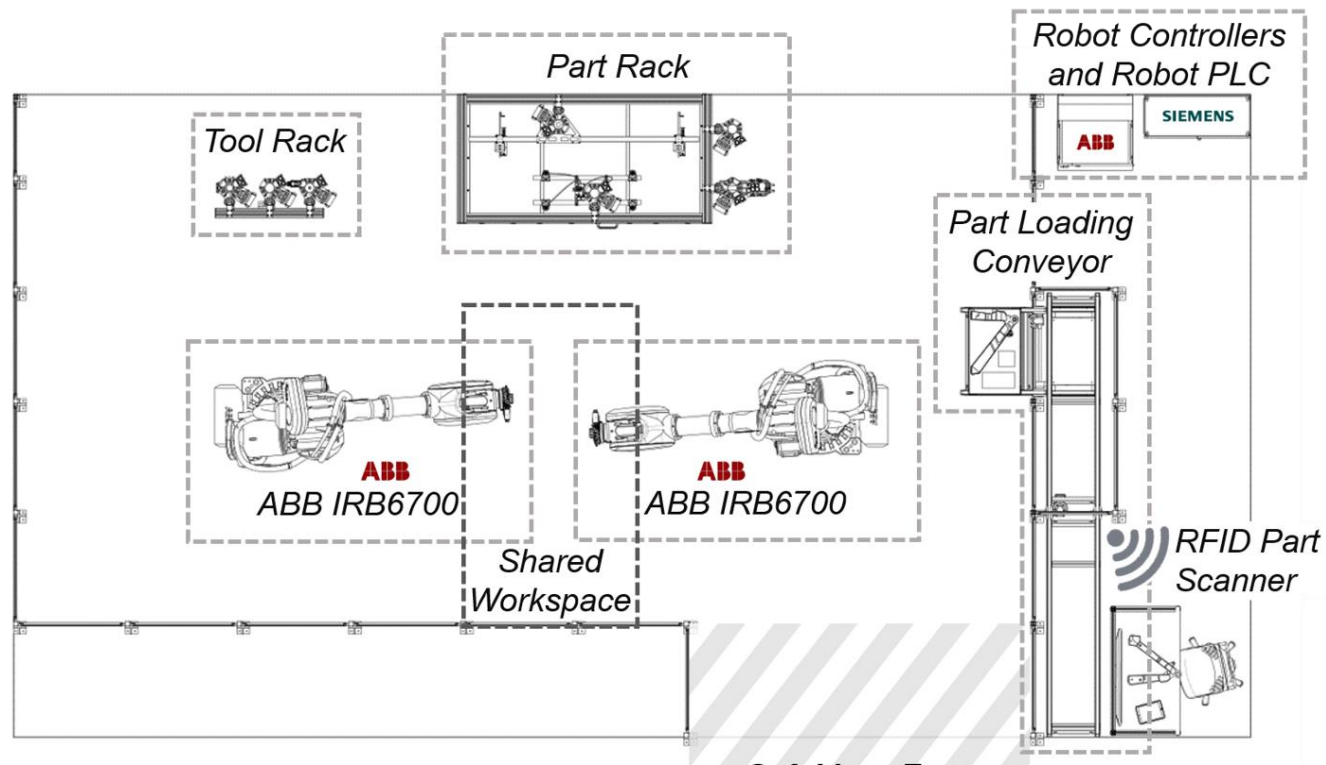

SafeMove Zone

Figure A 1. Schematic view of the FA3D-ABB cell layout.

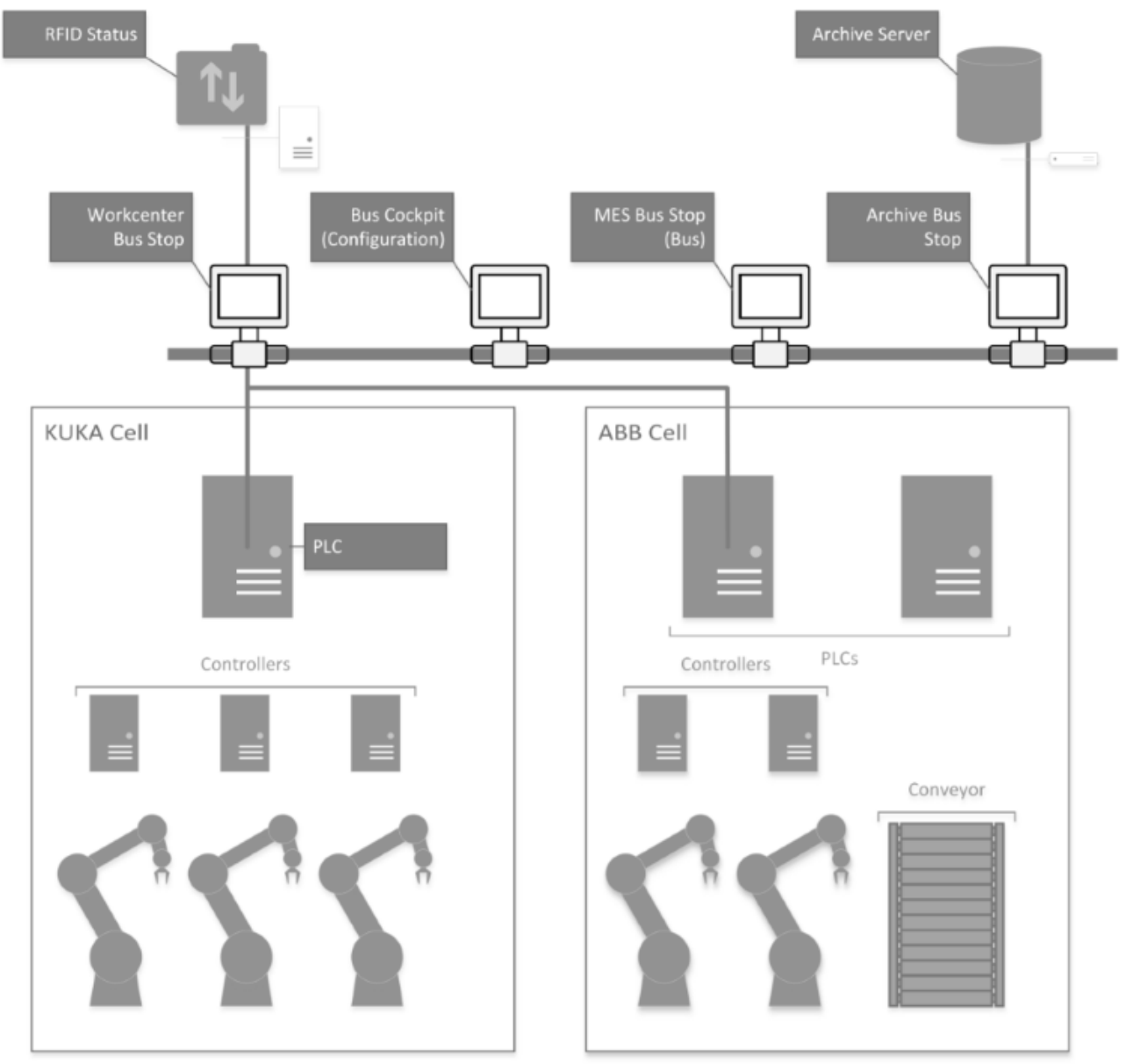

Figure A 2. The combined FA3D control network (excluding metrology detail).

Page 9 of 10 


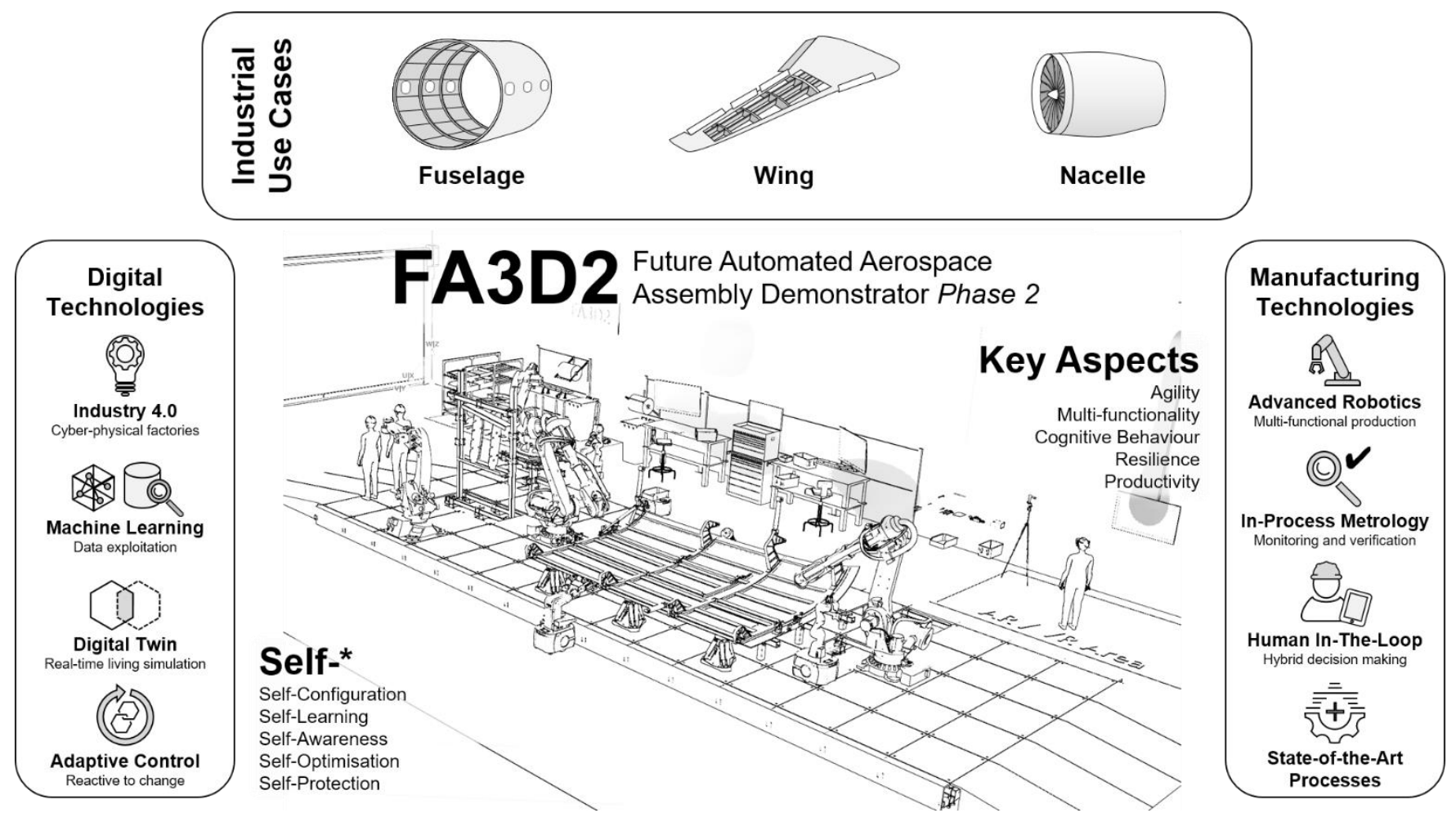

Figure A 3. FA3D2 Concept Overview.

Table A 1. Summary of Evolvable Assembly Systems demonstrators.

\begin{tabular}{|c|c|c|c|}
\hline Demonstrator & Concepts / Applications & Production processes & Products/domains \\
\hline SMART [46] & $\begin{array}{l}\text { - Batch size of one } \\
\text { - Disruption response } \\
\text { - Modular production systems } \\
\text { - Agent-based control and routing } \\
\text { - Recipe-based production } \\
\text { - Enhancing legacy systems }\end{array}$ & - Discrete constrained processes & $\begin{array}{l}\text { - Customised/personalised food and } \\
\text { pharmaceuticals }\end{array}$ \\
\hline PAD [54] & $\begin{array}{l}\text { - Batch size of one } \\
\text { - Plug and produce } \\
\text { - Agent-based planning and control } \\
\text { - Recipe-based production }\end{array}$ & - Robotic assembly & $\begin{array}{l}\text { - Consumer products } \\
\text { - Automotive / aerospace } \\
\text { subcomponents (e.g. truck glove box } \\
\text { hinge) }\end{array}$ \\
\hline FA3D-ABB [55] & $\begin{array}{l}\text { - Batch size of one } \\
\text { - Flexible and reconfigurable } \\
\text { manufacturing system } \\
\text { - Recipe-based production } \\
\text { - Agent-based control } \\
\text { - Agent-based planning } \\
\text { - Plug and produce } \\
\end{array}$ & $\begin{array}{l}\text { - Robotic inspection } \\
\text { - Human-robot interaction } \\
\text { - RFID part ID }\end{array}$ & - Aerospace components \\
\hline $\begin{array}{l}\text { FA3D-KUKA } \\
{[56]}\end{array}$ & $\begin{array}{l}\text { - Batch size of one } \\
\text { - Transformable manufacturing system } \\
\text { - Recipe-based production } \\
\text { - Agent-based control }\end{array}$ & $\begin{array}{l}\text { - Accurate robotic assembly (positioning, } \\
\text { drilling, rivetting) } \\
\text { - Low-cost reconfigurable fixturing } \\
\text { - Large volume metrology } \\
\text { - RFID part location and tracking }\end{array}$ & - Aerospace products \\
\hline FA3D2 & $\begin{array}{l}\text { - Transformable and scalable } \\
\text { manufacturing system }\end{array}$ & $\begin{array}{l}\text { - Large-scale accurate robotic assembly } \\
\text { (positioning, drilling, rivetting) } \\
\text { - Reconfigurable fixturing } \\
\text { - Large-volume metrology } \\
\text { - Including large and small scale robotics }\end{array}$ & - Aerospace products \\
\hline
\end{tabular}

